\section{Successful treatment of diffuse lipoid pneumonitis with whole lung lavage}

\author{
Han-Yu Chang, Chang-Wen Chen, \\ Chiung-Yu Chen, Tzuen-Ren Hsuie, \\ Cheng-Ren Chen, Wu-Wei Lei, \\ Min-Ho Wu, Ying-Tai Jin
}

\begin{abstract}
Diffuse lipoid pneumonitis is rare. Prednisolone can be beneficial, but no other method of treatment has been tried. The first case of diffuse lipoid pneumonitis successfully managed with whole lung lavage is described.
\end{abstract}

(Thorax 1993;48:947-948)

Whole lung lavage, first described by Ramirez in $1965,{ }^{1}$ is the most effective treatment of symptomatic pulmonary alveolar proteinosis. ${ }^{2}$ Occasionally it may be beneficial in patients with alveolar microlithiasis, radioactive dust inhalation, cystic fibrosis, or refractory asthma. ${ }^{23}$ However, clinical indications for whole lung lavage are still limited. ${ }^{3}$ Recently we successfully used whole lung lavage in the treatment of diffuse lipoid pneumonitis with both radiological clearing and improved pulmonary function after six months.

\section{Case report}

A 36 year old woman was admitted with two weeks of progressive exertional dyspnoea. She was a non-smoker and had been well until three months before admission. A nonproductive cough occurred without fever, wheezing, orthopnoea, or nocturnal dyspnoea. Her past history was unhelpful.

Physical examination showed a tachypnoeic woman with a shallow respiratory pattern and central cyanosis. Mild finger clubbing was present but there was no hepatosplenomegaly or ankle oedema. Cardiac examination was normal. Chest auscultation revealed inspiratory crackles in both middle and lower lung fields. Chest radiography showed bilateral diffuse alveolar shadowing, especially over the middle and lower zones (fig 1). Arterial blood gas tensions on air were $\mathrm{PO}_{2} 44.4 \mathrm{~mm} \mathrm{Hg}, \mathrm{PCO}_{2} 32.8 \mathrm{~mm} \mathrm{Hg}$,

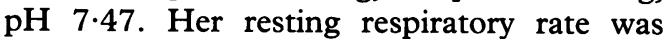
28/minute and her full blood count and blood chemistry were within normal limits. Pulmonary function tests performed upon admission gave an FVC of 2.101 (77\% pre- dicted), and $\mathrm{FEV}_{1}$ of 1.591 (63.1\% predicted), TLC $2.961 \quad(74.2 \%$ predicted). Single breath carbon monoxide diffusing capacity was not performed as she could not hold her breath for 10 seconds.

Methylprednisolone, $120 \mathrm{mg}$ per day, was started on the basis of a clinical diagnosis of diffuse pneumonitis, but an open lung biopsy performed one week later confirmed a lipoid pneumonitis without fibrosis (fig 2). Upon further questioning she admitted that one year ago she had been a religious fanatic and had taken $200-500 \mathrm{ml}$ coconut oil each day in order to purge her heart of sin through emesis and diarrhoea.

Whole lung lavage was contemplated after two weeks of high dose corticosteroids as her dyspnoea and chest radiographic findings remained the same. Arterial blood gas tensions just before whole lung lavage, with oxygen at $41 / \mathrm{min}$ via nasal prongs, were $\mathrm{Po}_{2}$ $66 \cdot 2 \mathrm{~mm} \mathrm{Hg}, \mathrm{PCO}_{2} 37 \cdot 3 \mathrm{~mm} \mathrm{Hg}, \mathrm{pH} 7 \cdot 46$.

Her resting respiratory rate was $28 /$ minute. She was premedicated with diazepam $20 \mathrm{mg}$ intravenously, pethidine $50 \mathrm{mg}$, and atropine $0.5 \mathrm{mg}$ intramuscularly and a $35 \mathrm{Fr}$ Bronchocath endobronchial tube was inserted under bronchoscopic guidance. She was ventilated with $100 \%$ oxygen through a volume cycled ventilator (Servo 900C); pancuronium bromide $(0 \cdot 1 \mathrm{mg} / \mathrm{kg})$ was given to achieve total paralysis. No anaesthetic agents were used. We first clamped the channel to the left

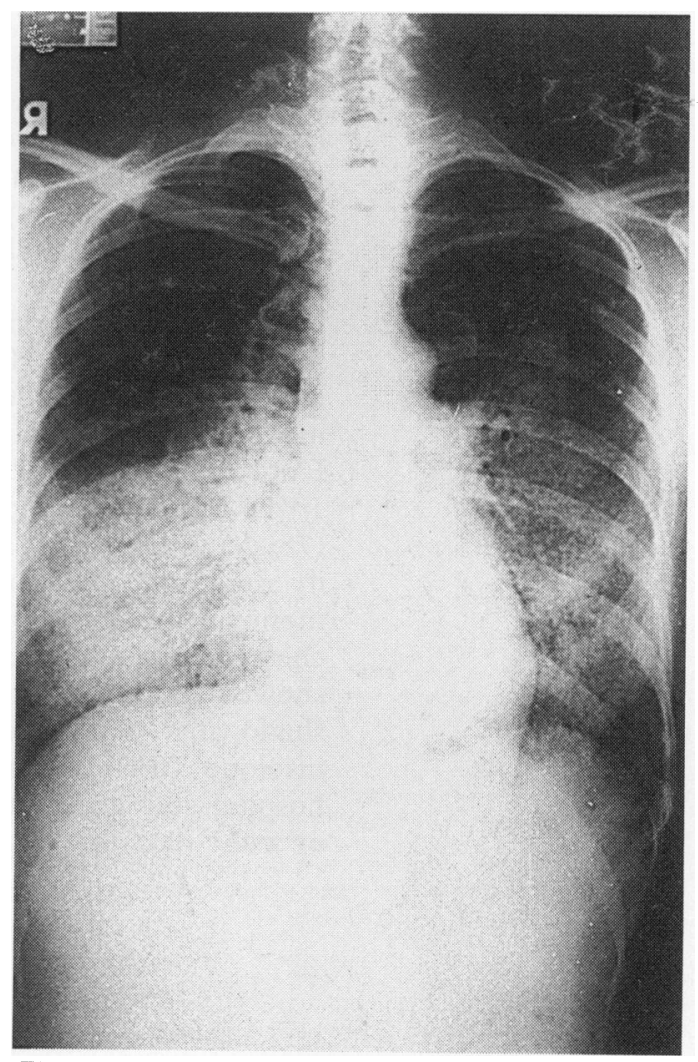

Figure 1 Chest radiograph showing bilateral diffuse alveolar shadow, especially over the middle and lower lung alveolds. 


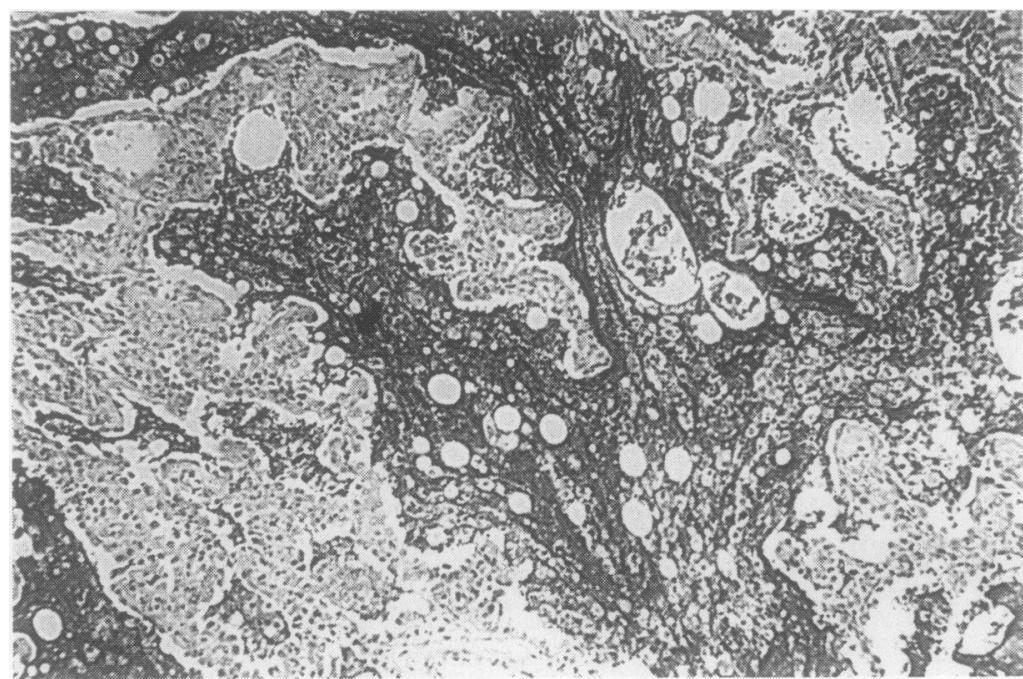

Figure 2 Open lung biopsy showing lipid filled macrophages and scattered lipid droplets in the necrotic foci. Haematoxylin and eosin. lung and allowed five minutes for degassing. Thereafter we started to fill the left lung with warmed isotonic saline $\left(37^{\circ} \mathrm{C}\right)$ by gravity from a bottle $30-35 \mathrm{~cm}$ above the patient. After the lavage fluid ceased to enter the lung, drainage was accomplished immediately by postural drainage aided with low pressure suction (10-20 mm Hg) as well as chest wall percussion. The aspirated fluid showed yellowish oil floating on the warm saline. This procedure was repeated until the drainage fluid looked clear. The size of each aliquot ranged from $250 \mathrm{ml}$ to $700 \mathrm{ml}$. Volumes delivered and recovered during left lung lavage were $9255 \mathrm{ml}$ and $8340 \mathrm{ml}$ respectively. Her arterial blood gas tensions after the lavage, on $40 \%$ oxygen from a Venturi mask, were $\mathrm{Po}_{2} 133.6 \mathrm{~mm} \mathrm{Hg}, \mathrm{PCO}_{2} 37.8$ $\mathrm{mm} \mathrm{Hg}, \mathrm{pH} 7 \cdot 44$. A right sided whole lung lavage was performed three days later; the volumes delivered and recovered were 8700 $\mathrm{ml}$ and $7930 \mathrm{ml}$ respectively. She tolerated the procedure well and her dyspnoea improved markedly shortly after. Arterial blood gas tensions on air were $\mathrm{Po}_{2} 73.8 \mathrm{~mm}$ $\mathrm{Hg}, \mathrm{PCO}_{2} 38 \cdot 2 \mathrm{~mm} \mathrm{Hg}$, pH 7.45. Her resting respiratory rate was $20 /$ minute. Repeated pulmonary function test on discharge showed an FVC of 2.021 ( $73.7 \%$ predicted), $\mathrm{FEV}_{1}$ of $1.811(71.8 \%$ predicted), and TLC 3.121 (78.2\% predicted). The lung diffusing capacity determined by the single breath carbon monoxide method was $38 \cdot 3 \%$ predicted. A chest radiograph taken just before discharge showed almost complete clearance of alveolar shadowing. She received high dose prednisolone (60-120 mg/day) throughout her hospital stay and this was gradually tapered over the next three months. Both pulmonary function test and chest radiographs at follow up showed improvement. After six months the diffusing capacity was $99.5 \%$ of predicted with normal spirometry and lung volumes and a normal chest radiograph.

\section{Discussion}

Pneumonitis due to the aspiration of oil (exogeneous lipoid pneumonitis) has been known for many years and most cases were attributable to the medicinal use of mineral and cod liver oil. ${ }^{4}$ It is an uncommon disorder. ${ }^{5}$

The reasons why oils could be aspirated into the lung include depressed cough mechanism, persistent vomiting, damaged tracheobronchial ciliary movement, or the bland nature of the inhaled oil. ${ }^{6}$ The types of reaction within the lung depend on the chemical nature of the oil. Mineral oil and vegetable oil are inert unless they are retained in the lung for a long time, while animal oils are hydrolysed into fatty acids by lipase and can produce an intense inflammatory reaction. ${ }^{7}$

Pulmonary involvement in our patient was distinguished by its diffuse pattern, which is only rarely reported. ${ }^{8}$ Ayvazian et al reported two cases of diffuse lipoid pneumonitis in whom prednisolone alone was used with success. ${ }^{8}$ However, their patients were only mildly hypoxaemic and the response to prednisolone occurred early. The lack of reponse to high dose corticosteroid therapy and the minimal fibrotic changes in the lung biopsy, which may be the result of the inert nature of coconut oil, helped in our decision to perform whole lung lavage. The subsequent dramatic relief of dyspnoea, accompanied by improved oxygenation and radiological clearing, is likely to be the result of removal of the oil from the alveolar spaces. Although corticosteroid therapy may have contributed to the continuing improvement in both pulmonary function tests and the chest radiographs, the lung lavage formed the cornerstone of treatment in this patient.

1 Ramirez RJ, Kieffer RF Jr, Ball WC Jr. Bronchoalveolar

lavage in man. Ann Intern Med 1965;63:819-28.
2 Alfrey DD, Benumof JL, Spragg RG. Anesthesia for bronchopulmonary lavage. In: Kaplan JA, ed. Thoracic anes- N thesia. New York: Churchill Livingstone, 1983:403-19. N

3 Reynolds HY. Bronchoalveolar lavage. Am Rev Respir Dis C 1987;135:250-63.

4 Foe RB, Bigh RS Jr. Lipoid pneumonia following occupational exposure to oil spray. $₹ A M A 1954 ; 155: 33-4$.

5 Wheeler PS, Stitik FP, Hutchins GM, Klinefelter HF, Siegelman SS. Diagnosis of lipoid pneumonia by com- ? puted tomography. FAMA 1981;245:65-6.

6 Graef I. Pulmonary changes due to aspiration of lipids and mineral oil. Am ₹ Pathol 1935;11:862-3.

7 Pinkerton $\mathrm{H}$. The reaction to oils and fats in the lung. Arch Pathol Lab Med 1928;5:380-401.

8 Ayvazian LF, Steward DS, Merkel CG, Frederick WW. Diffuse lipoid pneumonitis successfully treated with prednisolone. Am F Med 1967;43:930-4. 